\title{
Linx
}

Revue des linguistes de l'université Paris X Nanterre

$51 \mid 2004$

Théories de l'écriture et pratiques scolaires

\section{Brouillons scolaires et critique génétique : nouveaux regards, nouveaux égards?}

\section{Claudine Fabre-Cols}

\section{(2) OpenEdition}

\section{Journals}

Édition électronique

URL : http://journals.openedition.org/linx/160

DOI : $10.4000 / \operatorname{lin} x .160$

ISSN : 2118-9692

Éditeur

Presses universitaires de Paris Nanterre

\section{Édition imprimée}

Date de publication : 1 décembre 2004

Pagination : 13-24

ISSN : 0246-8743

\section{Référence électronique}

Claudine Fabre-Cols, «Brouillons scolaires et critique génétique : nouveaux regards, nouveaux

égards ? », Linx [En ligne], 51 | 2004, mis en ligne le 28 janvier 2011, consulté le 30 avril 2019. URL:

http://journals.openedition.org/linx/160 ; DOI : 10.4000/linx.160 


\title{
Brouillons scolaires et critique génétique : nouveaux regards, nouveaux égards?
}

\author{
Claudine Fabre-Cols \\ IUFM de Grenoble - LIDILEM Grenoble III - EA 609
}

À partir du moment où les travaux de la critique génétique ont été connus en didactique de l'écriture, la production d'écrits en contexte scolaire, dans son ensemble, a bénéficié d'une considération plus attentive et plus réaliste. C'est du moins ce que voudrait soutenir la présente intervention, que j'aimerais situer par quelques remarques liminaires.

Les brouillons: si on les considère sous l'angle du changement, on pense bien sûr à leur récente émergence comme objets didactiques. Les brouillons ne sont plus vus seulement comme des outils routiniers, des palliatifs marginaux, nécessaires surtout aux «mauvais » scripteurs, ils commencent à exister du point de vue didactique (vers 1985 selon C. Boré, Pratiques, juin 2000). Des facteurs venus tant de la recherche que de l'institution les rendent légitimes comme témoins et moteurs d'un travail langagier et textuel dont ils sont le lieu le plus visible.

Dès lors, on peut les définir comme des instruments de constitution ou de relance $d u$ processus rédactionnel, et leur reconnaitre une place centrale dans la formation du sujet locuteur-scripteur.

Ce qu'a changé la critique génétique: une influence n'étant pas seule en cause, il semble plus juste de dire ce qui a changé à partir du moment où les travaux de la critique génétique ont commencé à être connus à l'école, et de distinguer entre les évolutions des recherches et ce qu'en font dans leurs pratiques les enseignants et les élèves. Il est hélas bien clair que tout apport de la recherche n'ouvre pas directement sur des interventions pédagogiques et didactiques pertinentes, comme le montrent, depuis les années 70 , nombre d'avancées théoriques non suivies d'effets massifs sur le terrain (voir A. Petitjean, Colloque Inrp, mars 2002). En outre, s'agissant d'écriture, on sait que l'énonciation en terrain scolaire est soumise à des impératifs contradictoires (S. Plane, 
Colloque Inrp, mars 2002), ce qui contribue encore à compliquer la traçabilité de phénomènes en eux-mêmes fugaces.

Quant au domaine de recherche ici représenté par l'Item, il a d'abord été dénommé manuscriptologie (1972-82) puis quelquefois linguistique génétique, ou plus récemment génétique des textes (P.-M. de Biasi, Nathan, 2002), ou encore critique génétique (A. Grésillon, Eléments de critique génétique. Lire les manuscrits modernes PUF, 1994 ; M. Contat \& D. Ferrer, Pourquoi la critique génétique? Cnrs éd 1998)

Même si leurs variations peuvent devenir négligeables une fois défini un champ de recherche particulier, ces appellations méritent qu'on s'y arrête dans un premier temps, non seulement parce qu'elles renvoient à l'histoire de plusieurs groupes, et à la formation de notions et de méthodes, mais surtout parce qu'elles ont pu focaliser leur intérêt sur des points distincts, bien que proches, certaines semblant renvoyer plutôt à des corpus littéraires, voire aux spécificités du faire d'un auteur, d'autres renvoyant aussi bien aux textes littéraires qu'aux non littéraires, et s'intéressant explicitement aux phénomènes généraux de la production scripturale ${ }^{1}$. Certes, comme cela a déjà été souligné, il s'agit, à travers ces désignations, de tonalités et non de différences fortes, mais dans la pratique d'une recherche, l'accent peut, à travers elles, se déplacer du plus général au plus spécifique.

Ceci étant posé, l'analyse des brouillons scolaires doit sans cesse traiter de deux problèmes-clés :

- quels sont les événements scripturaux qui reviennent souvent pour tel scripteur particulier à tel moment de son histoire ? Quels sont ses rituels et ses modes d'écrire particuliers (entendons par là : ceux qui lui sont utiles à un moment donné) ? Etudier ces questions à l'école permettrait d'approfondir les spécificités de chacun ;

- qu'est-ce qu'écrire? Quelles sont les composantes de toute production écrite? Quelles sont les manières de composer, d'inscrire, de se relire, que l'on peut observer chez toutes sortes de scripteurs? Ces questions-ci, tout aussi urgentes à l'école, s'abordent plutôt en mettant l'accent sur des invariants (des sortes d'universaux ?) de l'activité scripturale.

C'est donc à la fois de critique génétique et de génétique des textes qu'il sera question ici, et je me réfèrerai essentiellement à leurs fondements linguistiques ${ }^{2}$.

Je reformulerai donc ainsi mon propos : qu'est-ce qui, dans la réflexion et dans les pratiques en didactique de l'écriture, a commencé à changer quand les travaux de la critique génétique ont été connus en ce domaine, c'est-à-dire à partir de 1983-84 ? (Je renvoie ici à mes publications de 1983, 1987, 1990).

Cette influence d'un domaine d'étude à un autre me semble particulièrement sensible selon trois axes : la critique génétique a proposé un ancrage théorique et une

\footnotetext{
${ }^{1}$ Ces termes peuvent aussi s'entendre diversement, ainsi qu'il a été dit lors du colloque de mai 2003, ce qui ne saurait surprendre étant donné l'extension variable du terme texte, et les changements pouvant se produire de génétique adjectif à génétique nom.

${ }^{2}$ Cet article se présente sous une forme très proche des notes qui ont soutenu mon intervention au colloque de Nanterre en mai 2003. Comme celle-ci, il s'adresse à des didacticiens familiers des questions de réécriture, à qui j'ai pensé pouvoir épargner certaines redites! Les définitions et les exemples peuvent être retrouvés dans la bibliographie.
} 
méthode d'analyse des activités d'écriture; elle a permis de (se) représenter le fait d'écrire comme un travail et une recherche; elle a placé le sujet scripteur au centre de sa production.

\section{Un ancrage théorique et une méthode d'analyse des activités d'écriture}

Rendant compte de la genèse des textes écrits à la lumière des théories de l'énonciation telles que les formule Emile Benveniste, les chercheurs en critique génétique montrent qu'il existe des composantes générales de toute production écrite, en tant qu'elle est une production langagière.

Ils proposent également, et illustrent, une méthode d'analyse des différents états d'un texte (soit des notes, plans et avant-textes manuscrits ou tapuscrits jusqu'à l'écrit publié) qui peut être transposées aux brouillons et copies d'écoliers.

\section{I.1. L'ancrage théorique est constitué par les théories de l'énonciation et renvoie aux travaux d'E. Benveniste (Problèmes de linguistique générale, 1966 et 1974), A. Culioli et C. Fuchs. Deux points sont particulièrement mis en valeur:}

a) L'«énonciation à l'état naissant» (A. Grésillon \& J.-L. Lebrave, Langages, n 69 , 1983 ) est manifestée matériellement par des traces perceptibles dans les brouillons et manuscrits. À travers leur observation, analyse, classement et interprétation, il est possible d'accéder en partie à l'histoire et à la compréhension des événements langagiers dont des traces lisibles sont conservées. A travers le texte final sont perçus des textes virtuels, des possibles envisagés.

b) La notion de "polyphonie énonciative » (Langages, $\mathrm{n}^{\circ}$ 69, 1983) précise les rôles du sujet dans la production écrite (il est scripteur et lecteur alternativement). «Dès la première rature, le manuscrit est le lieu d'une confrontation permanente entre écrire et lire » (P.-M. de Biasi, 1994), la lecture étant un temps d'activité métalinguistique et métadiscursive (J. Rey-Debove, 1982) qui accompagne ou encadre les moments de scription. Les chercheurs désignent ces deux types d'activité par le terme de «double locution ». Les modifications (ou ratures) inscrites dans les brouillons et manuscrits correspondent ainsi à un énoncé métalinguistique toujours possible, non-dit, marqué plus ou moins clairement.

\section{2. Une méthode d'analyse}

Les traces des activités de production écrite telles qu'elles sont conservées dans les brouillons et manuscrits deviennent, grâce aux travaux de l'Item et de ses collaborateurs, des phénomènes observables, classables et analysables sur la base de méthodes linguistiques.

a) En listant les énoncés substituables qui peuvent être reconstitués en un point du texte, les chercheurs dressent des paradigmes par lesquels deviennent visibles les hésitations, les bifurcations envisagées par le scripteur au moment même où il écrit. Par exemple, à propos de quelques lignes de Proust (voir La genèse du texte: les modèles linguistiques), Catherine Fuchs met en évidence, à travers les divers états du manuscrit, 
des familles d'énoncés unis par une relation de paraphrase, ce qui montre bien que certains thèmes ou images insistent, apparaissant sous formes différentes et mouvantes, jugées semble-t-il insatisfaisantes tant qu'elles sont reprises, jusqu'au point où quelque chose se fixe pour le scripteur dans certains termes, certains rythmes, rendant acceptable ce qui est déjà tracé, et faisant qu'une suite peut être réalisée.

b) Ces énoncés modifiés sont aussi analysables selon des ensembles syntagmatiques, et la progression d'un état de texte à un autre peut être reconstituée selon des opérations linguistiques de suppression, d'ajout, de déplacement, de permutation, de remplacement, c'est-à-dire de substitution sous diverses formes ${ }^{3}$.

\section{3. Incidence de ces deux points sur les pratiques scolaires}

a) Tout d'abord, l'idée qu'il y a du connaissable dans ce domaine semble avoir progressé : des enseignants et des élèves, puis des instructions officielles et des manuels, admettent que la démarche d'écriture laisse des traces, qu'une part des phénomènes de textualisation est reconnaissable lorsqu'ils se produisent dans le travail du brouillon, ou repérable après coup, qu'il existe des modifications manifestement insatisfaisantes pour le scripteur comme pour les lecteurs et des points d'équilibre perceptibles. D'où, à partir de là, des possibilités d'intervention didactique raisonnée. Certes, préparer, accompagner ou évaluer une pratique ne garantit pas une efficacité absolue. Toutefois, ce brin de rationalité a peut-être rendu enseignants et élèves moins fatalistes, moins désarmés en la matière lorsque le «don » est supposé faire défaut.

b) Que toute production écrite est d'abord une production langagière, cela ne figure pas toujours dans les attendus de l'activité scripturale à l'école : l'ancrage théorique cidessus comble à l'évidence une lacune. Dès lors, on peut stimuler cette production avec les moyens qui sont convenables pour les productions orales, la comprendre et la relancer également dans et par l'intertextualité (voir les publications de J. AuthierRevuz et les divers travaux concernant «l'oral dans l'écrit » à l'école, en particulier N. Marty 1991).

On peut aussi rattacher indirectement à cette prééminence des théories énonciatives l'idée que, si la maîtrise rhétorique des discours peut être en rapport avec un degré de culture qui requiert du temps, la dynamique énonciative, elle, en tant que besoin expressif du sujet, n'a pas d'âge. C'est pourquoi il ne m'a jamais semblé juste de considérer les brouillons du primaire comme le lieu où domineraient les modifications et apprentissages formels, tandis que les écrits du collège et du lycée témoigneraient mieux de l'énonciation scripturale.

c) Les deux points précédents ont encouragé un retour à l'observation des brouillons en tant que témoignages des processus de production scripturale, un souci des textes concrets, observation que minore quelquefois le recours à des modèles $a$ priori. Depuis les années 1985 se sont maintenues et développées les recherches sur des corpus diversifiés (concernant les situations et les contextes, les consignes, les oraux

\footnotetext{
${ }^{3}$ Le remplacement correspond à la forme la plus évidente de la substitution; l'ajout et la suppression correspondent chacun à une substitution dont l'un des termes est nul, tandis que le déplacement combine, en deux points de l'axe syntagmatique, un ajout et une suppression.
} 
de travail, les brouillons et copies). Ainsi, au lieu de se focaliser sur la seule «copie au propre », l'observation a englobé documents et échanges préparatoires pour tenter d'interpréter le processus de production.

Le sens n'est pas tout entier au départ, il est un produit, c'est ce que montrent les reconstitutions génétiques effectuées sur les manuscrits d'écrivains. Nul doute que la publication des hypothèses concernant la démarche d'écriture de ces scripteurs exceptionnels a revalorisé tous les écrits, et en particulier les écrits scolaires.

d) Tout ceci a-t-il affaibli la réception normative, voire surnormative, qui est dominante à l'école ? Se rendre attentif aux processus d'écriture devrait permettre, à l'enseignant comme à l'élève, de ne pas focaliser la réception sur les «fautes", mais plutôt sur la recherche dont les modifications constituent l'indice, et d'apprécier, entre autres, les problèmes résolus, les démarches individuelles. Pour l'élève, cela signifierait qu'il s'estime autorisé aux essais et erreurs, aux tâtonnements et à la recherche. Pour l'enseignant, cela signifierait qu'à côté de son rôle normatif indispensable, il adopte une attitude plus ouverte, d'observation et d'interprétation. Lisant les textes d'enfants comme on peut lire des manuscrits d'écrivains, un tel enseignant serait susceptible d'accompagner l'écriture comme processus, et pas seulement capable de juger d'un texte final. Il semble que nous n'en sommes pas là.

L'évaluation, y compris l'évaluation officielle, a cependant évolué. Elle ne concerne plus seulement le produit (encore que...), mais peut porter sur les modalités de la production (construire des compétences, prendre conscience d'un parcours, le modifier sur tel ou tel point). Quant à l'auto-évaluation, elle induit ou inclut des découvertes proches de la double énonciation dont il a été question plus haut.

\section{II. (Se) représenter toute écriture comme un travail et une recherche}

La notion même de genèse met au premier plan celle d'une production malléable, entre autres, selon le temps qu'on lui consent. L'observation de manuscrits d'écrivains atteste qu'en composant, les plus fameux d'entre eux ont hésité, cherché, quelquefois renoncé, comme le font les élèves et les adultes "tout venant». Cette observation bouleverse les représentations sacralisantes de l'écriture : elle montre celle-ci comme un travail qui ne va le plus souvent pas sans blocages et reprises, à travers des modifications tâtonnantes chez les scripteurs exceptionnels comme chez les autres.

II.1. Quel enseignant, depuis une quinzaine d'années, n'a pas tenté l'expérience : se procurer la copie d'un avant-texte d'écrivain, en faire un transparent, le projeter devant sa classe, et se voir, le plus souvent, agréablement surpris par les commentaires des élèves, ravis de ne pas être les seuls à peiner pour écrire, puisque Balzac, Flaubert ou Proust ont eux aussi hésité, raturé ? Découvrir des avant-textes d'écrivains revient alors à faire voir la production écrite sous un jour entièrement neuf, à déculpabiliser et à stimuler les essais et erreurs. Le choc ressenti met à mal les vues les plus naïves quant au don d'écriture, fait apparaître la récurrence des modifications de genèse, et l'existence ainsi légitimée d'états intermédiaires quelquefois peu satisfaisants, qui permettent d'accéder à un état final supposé l'être davantage.

II.2. Ces aperçus, bien que sommaires, lors de la lecture d'oeuvres littéraires (avant-textes à l'appui), ainsi qu'une pratique plus réflexive des productions effectuées en classe, font voir que l'écrit est transformable dans le temps. Ainsi peuvent être 
concrètement manipulées et appréciées les notions de variante, d'avant-texte, d'états de texte, de phases rédactionnelles, de dossier génétique. En conséquence, l'écriture apparaît aussi comme un travail. Les modifications n'ont pas un caractère infamant, elles ne correspondent pas toujours à des manques, mais peuvent constituer des indices d'une recherche. Loin d'être un raté, la rature semble plutôt une régulation fonctionnelle, pour peu que le scripteur dispose de temps et de stimulations.

II.3. L'observation d'avant-textes littéraires et de productions scolaires permet aussi de voir qu'il n'y a pas un moule unique du «bien-écrire ». Des procédures de textualisation très différentes s'observent, même parmi les scripteurs les plus « experts », en particulier dans l'opposition entre les modes d'écrire dits «à programme » et ceux dits "à processus », et dans leurs éventuelles intrications.

Sur quelles variables asseoir ces différences? Certains travaux de l'Item se proposaient de vérifier l'effet du type de texte sur le mode d'écrire d'un même scripteur. Plus tard, dans le sillage des travaux du groupe Eva-Inrp, beaucoup d'enseignants se sont efforcés d'évaluer l'impact des conditions de rédaction et des dispositifs didactiques, et aussi bien sûr du type de texte, sur les productions des élèves. Plus récemment encore, on a pu envisager le mode d'écrire comme une tendance individuelle, les types de scripteurs scolaires se distinguant en «planificateurs» ou non, «progressifs» ou non, «inséreurs ou chambouleurs » (contributions de D. Bessonnat, Pratiques n¹05-106).

Il y là des pistes qui méritent d'être encore explorées. En effet, si les pratiques scolaires ont plutôt bien intégré les changements de représentations induits par la vulgarisation des recherches en faisant lire les textes classiques auprès de leurs avanttextes, pratiques courantes au collège et au lycée, en revanche, du côté de la production scolaire, c'est surtout à l'école élémentaire que quelques mises en oeuvre ont été tentées.

\section{Placer le sujet scripteur au centre de sa production}

En cela la critique génétique rejoint d'autres orientations influentes en didactique, mais elle montre du sujet une zone inaccessible autrement, celle de son énonciation scripturale.

De façon dominante à l'école (voir I.O. et manuels), le sujet scripteur est supposé identique à l'élève, c'est-à-dire défini par sa progression dans la maîtrise des normes, habiletés et savoir-faire scripturaux.

Complémentairement, l'observation des brouillons et copies permet de repérer les marques d'une démarche plus personnelle: procédures individuelles, rituels idiosyncrasiques (tendance à la suppression / à la refonte / à l'ajout...), activités épilinguistiques et métalinguistiques, présence et qualités de l'auto-évaluation, n'apparaissent plus seulement estimables et didactisables selon la qualité du produit final.

III.1. La critique génétique a ouvert la voie à l'observation et à la légitimation de manières d'écrire spécifiques, individuelles

a) Chez les scripteurs exceptionnels, en premier lieu: l'examen détaillé de dossiers génétiques, tout comme la lecture, vulgarisée et facilitée par la publication, de Carnets d'écrivains (entre autres, Flaubert, Zola, Valéry) montrent combien la diversité domine les habitudes de composition. 
Tel éprouve la nécessité absolue d'avoir le titre définitif de son écrit avant de commencer, tandis qu'un autre le trouvera seulement après la dernière ligne, et que tel autre encore en essaiera plusieurs en cours de route ; certains ne peuvent se mettre au travail avant d'avoir tout ou partie de leur plan, et d'autres se sentiraient bridés d'en avoir un ; l'impression que quelqu'un a déjà traité leur thème entraîne pour quelques scripteurs de la réticence, voire de l'impuissance, tandis que Montaigne et bien d'autres s'appuient avec bonheur sur toutes les formes de la citation et de l'emprunt.

Les auxiliaires et rituels diffèrent à l'évidence jusque dans leur matérialité : notes mentales dont il ne reste rien, carnets ou non, fiches, petites ou grandes feuilles, manuscrit ou tapuscrit, crayons ou encres, repères de couleurs ou de chiffres, schémas..., comme si tel écrivain éprouvait plus d'aisance et de créativité dans ce rituel particulier, de sorte qu' " une technique de travail, une démarche, un instrument... » peut quelquefois se retrouver "à l'échelle de son oeuvre entier» (P.- M. de Biasi, 1990).

Alors, à chacun sa façon d'écrire, à l'école aussi ? Ce n'est évidemment pas possible dans l'absolu, chaque scripteur y étant situé au sein de groupes, mais la question mérite d'être posée.

b) Hétérogénéité et spécificité des modes d'écrire à l'école

Depuis de nombreuses années, on admet que les sujets sont plus ou moins sensibles à certains éléments inducteurs en matière de mémoire (il y a les plutôt « visuels », les «auditifs »...). De même, s'agissant de la lecture, des recherches montrent qu'il existe des apprenants plutôt sensibles aux éléments sémiographiques (ces enfants sont dits «chinois»), d'autres plutôt sensibles aux éléments phonographiques (enfants dits " phéniciens »).

Qu'en est-il en matière d'écriture ? Peu de recherches existent ici en domaine français. On peut cependant citer, qui partent d'hypothèses psychologiques, les publications de M. Alcorta et d' A. Piolat ; qui partent des hypothèses de la génétique textuelle, outre mes propres travaux, ceux de C. Boré, J. David, et plus récemment C. Lacoste.

En contrastant deux suites rédactionnelles (notes, brouillons et texte final remis à l'enseignant) produites par deux écoliers de Cours moyen, dans la même classe et dans des conditions similaires, j'ai pu analyser deux manières de faire différentes, dominantes chez chacun : Isabelle s'efforce de textualiser dès le début de son travail, et même lorsqu'elle prend des notes ; puis elle recopie avec peu de modifications dans les brouillons suivants, et parvient dans le dernier état de texte à une version proche de la première ; au contraire, Stéphane prend des notes très succinctes, distingue nettement notations pour se souvenir et narration, travaille les différents états de son texte par la concision de certains détails et par l'amplification de l'ensemble, et modifie chaque version par rapport à celle qui précède. Il décrit ainsi un parcours de production qui va d'une sorte de script à des versions remaniées et de plus en plus développées (C. Fabre, 1987). Certes, il ne s'agissait là que d'une première approche, mais qui faisait bien apparaitre le fait que chaque scripteur, y compris dans le cadrage scolaire, pouvait tendre à privilégier certaines procédures plutôt que d'autres.

Les travaux de Catherine Boré (1998), puis ceux de Claire Lacoste (thèse de doctorat, Paris 3, 2002), s'appuyant à la fois sur des aspects longitudinaux et 
contrastifs, montrent à leur tour de façon fine que la diversité des modes d'écrire ne se rencontre pas seulement chez les écrivains. Les écoliers aussi, individuellement ou selon l'occasion, ont tendance à en privilégier certains. Selon Claire Lacoste, les deux principaux (accumulation et récurrence), ne sont pas sans rappeler l'écriture à programme et l'écriture à processus décrites chez les écrivains, tandis qu'un troisième mode, intermédiaire (dit du gonflement), participerait des deux autres.

A partir de productions par ordinateur et de l'enregistrement filmé des gestes d'écriture, cette conclusion confirme et approfondit celles des précédents travaux conduits avec des corpus scolaires manuscrits. Tous permettent d'approcher une définition du sujet scripteur construite non plus à partir des qualités académiques ou artistiques du texte qu'il a produit, mais par le mode d'écrire qu'il a, spontanément ou consciemment, déployé en écrivant.

\section{III.2. Un point de vue didactique: le sujet scripteur se construit dans l'intersubjectivité et l'intertextualité}

Subjectivité et intersubjectivité étant théoriquement liées (E. Benveniste), on peut s'attendre à ce que le sujet scripteur en formation se construise aussi dans l'altérité. La recherche en didactique a expérimenté des formes d'intersubjectivité propres au contexte scolaire ou à celui de la formation, par exemple les brouillons oraux à plusieurs voix ou les dictées à l'adulte.

En renvoyant à des pratiques scolaires qui s'appuient pour partie sur les théories déjà évoquées et pour partie sur une réflexion didactique originale (voir la thèse de Michèle Maurel, décembre 2002, Grenoble 3), on constate que :

a) l'intersubjectivité et l'intertextualité scolaires se fondent sur l'insertion des textes produits dans un contexte large, sur la mise en relation pragmatique des paroles dites ou écrites en classe avec des faits ou des discours situés dans un espace social compris hors les murs de la classe (correspondance, concours, publications, affiches, théâtre...) ; qu'elles se pratiquent dans la continuité entre parler et écrire, en particulier par le biais des avant-textes oraux (l'oral pour expliquer, argumenter, raconter, inventer), et sous les diverses formes maintenant bien décrites de la « rédaction conversationnelle ». Intertextualité et intersubjectivité renvoient aussi à la pratique de scripteurs supraindividuels, tantôt parce que la parole et l'écriture se font en petits groupes (lors des moments de recherche, de scription et de révision), tantôt par l'écriture à deux, en dyades de pairs ou en dyades inégales, en particulier lors de la dictée à l'adulte d'un texte composé par de très jeunes enfants.

b) L'intertextualité peut être entendue ici aussi bien au sens restreint (rapport d'un texte avec d'autres textes) qu'au sens large (entre autres, chaque texte suppose au moins deux sujets, celui qui l'émet et le groupe social dont il parle le langage). Dans la lignée des recherches de l'Item, elle renvoie à l'autodialogisme (discours métalinguistique virtuel du scripteur qui est son premier lecteur), au dialogisme entre le scripteur et le texte déjà là, à celui entre le scripteur et le récepteur virtuel qu'il suscite.

Et bien sûr, de façon littérale, elle ne cesse d'englober les liens tissés entre les textes, renvois, citations, pastiches, comparaisons, transformations, constructions de types. 
Des observations proprement didactiques tendent à montrer que dans un tel ensemble (intersubjectivité + intertextualité), le sujet scripteur a une chance de former sa propre griffe. De sorte que, si la critique génétique a légitimé la diversité des modes d'écrire, il semble que c'est plutôt la recherche en didactique de l'écriture qui a essayé de saisir la subjectivité scripturale à travers l'intersubjectivité manifeste.

\section{Conclusions}

Sous l'influence des recherches de l'Item et de leur adaptation au contexte scolaire, mais aussi de celles des groupes Inrp, on a vu en une vingtaine d'années les représentations cumulatives et normatives du savoir-écrire céder un peu de place à une conception plus ouverte, multidimensionnelle, de la production textuelle, qui prend en compte le temps, les dispositifs et les conditions de production, et la diversité des sujets-scripteurs.

Ainsi s'esquisse une orientation plus problématique de la didactique de l'écriture : il semble possible que ce ne soit plus seulement l'institution et la tradition qui fixent a priori ce qui doit être produit, ce que doivent être le produit et la production. Une grande part d'initiatives revient au sujet scripteur, qui peut appréhender et voir reconnue la spécificité de sa propre démarche, et l'auto-évaluer. Une grande part revient aussi à l'enseignant qui l'accompagne : écoute, observation, interrogation, attention aux spécificités individuelles aussi bien qu'à la transversalité des phénomènes. En ce sens, l'apport de la génétique textuelle a été fondamental et n'a pas fini de faire sentir son influence. Remarquons seulement que son caractère problématique et stimulant risquerait de ne pas résister dans un univers scolaire d'où la recherche serait bannie.

D'autre part, quel que soit le gain que représente la vulgarisation des travaux de l'Item, des questions proprement didactiques restent à approfondir dans le travail des brouillons en classe. Il s'agit entre autres de l'ouverture à des disciplines autres que l'enseignement de la langue et de la littérature, à tous les types textuels, à tous les supports (en particulier l'écran d'ordinateur) ou de la création de projets et de dispositifs de production généralisables. D'autre part, dans la pratique de l'enseignant, comment réaliser un juste équilibre entre la tâche d'amélioration du texte et celle de formation du sujet scripteur? Ou encore, comment pondérer l'acquisition des compétences transversales pour tous et le respect des rythmes et modes spécifiques de chacun? Le travail en petits groupes, l'intégration des avant-textes oraux et de l'autoévaluation demandent eux aussi encore davantage de recherches et de moyens.

Dans l'analyse et l'interprétation des oeuvres littéraires, la linguistique génétique est apparue dans un moment de crise, alors que les méthodologies l'ayant précédée (selon A. Grésillon, philologie, critique des sources, analyse structurale) ne paraissaient plus satisfaisantes. Dans la production et la réception des textes non littéraires, la génétique textuelle, de même que les autres domaines de recherche convoqués dans le colloque de Nanterre (mai 2003) apparaissent également à un moment où l'école et la société sont en crise. Ainsi, alors que des connaissances didactiques nouvelles, des expériences de qualité, accumulées, pourraient permettre de mieux enseigner, le moins qu'on puisse dire est qu'on ne constate pas d'amélioration massive sur le terrain. Que 
l'on pense, non seulement à l'adaptation des recherches de l'Item dont il est question aujourd'hui, mais aussi à l'extraordinaire effervescence d'enseignement et de recherche qui a donné le Plan Rouchette (1970) et s'est continuée jusqu'aux groupes Évaluation et Révision de l'Inrp, aux débats inlassables de la revue Pratiques, à la diffusion de modèles rédactionnels de toutes sortes, aux logiciels d'aide à l'écriture, pour ne citer que quelques-uns des facilitateurs dont on pourrait aujourd'hui disposer!

L'adaptation des travaux de la critique génétique a été un apport fondamental à cette richesse, et du point de vue méthodologique et parce qu'ils ont donné un regain de force problématique aux théories de l'énonciation. Le terrain scolaire a de son côté permis de développer certains des aspects spécifiques de l'intertextualité et de l'intersubjectivité. Aujourd'hui, ces différents apports restent fragiles et menacés : ils ne pourront fructifier que dans une école où la recherche vive. 
Brouillons scolaires et critique génétique...

\section{BIBLIOGRAPHIE}

ANIS, Jacques, LeBRAVE, Jean-Louis (éds.) (1991) Le texte et l'ordinateur : les mutations du lire-écrire, La Garenne-Colombes, Ed. de l'Espace européen.

BeLLEMIN-Noël, Jean (1972) Le texte et l'avant-texte, Paris, Larousse.

Benveniste, Emile (1966 et 1974) Problèmes de linguistique générale, t.1, t.2, Paris, Gallimard.

Bessonnat Daniel (éd.) Pratiques n 105-106, juin, 2000.

BIASI, Pierre-Marc de (1990) «Avant-texte », Le Grand Atlas Universel des Littératures, Encyclopedia Universalis France, p 25.

BIASI, Pierre-Marc de (1998) «Qu'est-ce qu'un brouillon? Le cas Flaubert : essai de typologie fonctionnelle des documents de genèse », in M. Contat et D. Ferrer (éds.), Pourquoi la critique génétique? éd. du CNRS, 31-60.

BORÉ Catherine, DAvid Jacques (1996) «Les différentes opérations de réécriture: des brouillons d'écrivains aux brouillons d'élèves", in Plane S. et Turco G. (éds), De l'évaluation à la révision, Paris, Hachette.

CONTAT Michel, Ferrer Daniel (éds.) (1998) Pourquoi la critique génétique? éd. du CNRS.

DAVID Jacques (1994) «Ecrire, c'est réécrire. De la pertinence des ratures chez l'écolier », Le français aujourd'bui $\mathrm{n}^{\circ} 108$.

FABRE Claudine (1982) «Dans la poubelle de la classe: subjectivité et jeux de langage », La linguistique, vol 18, fasc 2, Paris, PUF, 99-113.

FABRE Claudine (1983) «De quelques usages non-standard dans des écrits d'écoliers », in Frédéric François, J'cause français, non ?, Paris, Maspéro/La Découverte, 37-67.

FABRE Claudine (1987) Les activités métalinguistiques dans les écrits scolaires,Thèse de doctorat d'Etat, Sorbonne Paris 5.

FABRE Claudine (1991) «Brouillons d'écrivains Brouillons d'élèves », Madrid, Fuencarral.

FABRE Claudine (1991) «La linguistique génétique: une autre entrée dans la production d'écrits », Repères, INRP.

FABRE Claudine (1994) «Ce qui vaut pour un manuscrit d'écrivain vaut-il pour un brouillon d'écolier?", Le Français aujourd'bui, n 108.

FABRE Claudine (1990) Les browillons d'écoliers ou l'entrée dans l'écriture, Ceditel, Grenoble.

FABRE Claudine (éd.) (2000) Apprendre à lire des textes d'enfants, De Boeck Ed, Belgique.

FABRE Claudine (2002) Réécrire à l'école et au collège, ESF éd.

FuCHS Catherine \& al. (1982) La genèse du texte : les modèles linguistiques, éd. du Cnrs.

Garcia-Debanc Claudine (1990) L'élève et la production d'écrits, Metz.

GARCIA-DEBANC Claudine (1995) «La production d'écrits telle qu'on l'enseigne aujourd'hui », Etudes de linguistique appliquée, $\mathrm{n}^{\circ} 99$, Paris, Didier Erudition. 


\section{Claudine Fabre-Cols}

GRÉSILLON Almuth (1994) Eléments de critique génétique, Paris, PUF.

GrÉSILLON Almuth, Lebrave Jean-Louis (éds.) (1983) «Manuscrits Ecriture Production linguistique $"$, Langages $\mathrm{n}^{\circ} 69$.

GrÉSILLON Almuth, WernER Michel (1985) Leçons d'écriture, Paris, Minard.

Hay Louis, Nagy Peter (éds.) (1982) Avant-texte, Texte, Après-texte, Akademiai Kiado-éd. du CNRS, 1982

JAFFrÉ Jean-Pierre \& DAVID Jacques (1998) « Premières expériences en littéracie », Psychologie et éducation, 33, 47-61.

LACOSTE Claire (2003) Etude génétique de l'écriture sur traitement de textes d'élèves de CM2, année 199596, thèse pour le doctorat de Sciences du langage, Université de Paris 3

LAMOTHE-BORÉ Catherine (1998) Choix énonciatifs dans la mise en mots de la fiction: le cas des browillons scolaires, thèse pour le doctorat de Sciences du langage, Université StendhalGrenoble 3.

LEBRAVE Jean-Louis (1983) «Lecture et analyse des brouillons », Langages $\mathrm{n}^{\circ} 69$.

MARTY Nicole (1991) «L'écrit dans l'oral », Etudes de linguistique appliquée, nº 81.

MAUREL Michèle (2000) «De la copie à l'écrit personnel : formes et degrés de l'intertextualité en situation guidée », in Apprendre à lire des textes d'enfants, C. Fabre-Cols (Ed.), Bruxelles, De Boeck.

Piolat Annie (éd.) (1998) La rédaction de textes. Approche cognitive, Neuchâtel, Delachaux et Niestlé.

Plane Sylvie (1994) Ecrire au collège. Didactique et pratiques d'écriture, Paris, Nathan.

Plane Sylvie (2000) «Eléments pour un usage didactique du traitement de texte. Ecrire, réécrire et réviser sur ordinateur », Pratiques n¹05-106, 159-181.

ReY-Debove Josette (1982) "Pour une lecture de la rature », La genèse du texte: les modèles linguistiques, éd du Cnrs

REY-Debove Josette (rééd 1997) Le métalangage. Etude linguistique du discours sur le langage, Le Robert, Paris, A. Colin. 